PAPER

\title{
Isolated middle cerebral artery disease: clinical and neuroradiological features depending on the pathogenesis
}

\author{
P H Lee, S H Oh, O Y Bang, I S Joo, K Huh
}

J Neurol Neurosurg Psychiatry 2004;75:727-732. doi: 10.1136/jnnp.2003.022574

See end of article for authors' affiliations ....................

Correspondence to: Dr O Y Bang, Department of Neurology, School of Medicine, Ajou University, Woncheon-dong San 5, Paldal-Ku, Suwon, Kyungki-do 442-749, South Korea; phisland@ chollian.net

Received 4 July 2003 In revised form 23 September 2003 Accepted

25 September 2003

\begin{abstract}
Background: Isolated atherosclerotic middle cerebral artery (MCA) disease is often difficult to differentiate from cardioembolic disease if intracranial atherosclerosis coexists with cardiac disease.

Objectives: To evaluate whether clinical and neuroradiological features of isolated MCA disease differ according to the underlying aetiology.

Methods: Isolated MCA disease was defined as a unilateral angiographically occlusive lesion of the MCA on the symptomatic side without lesions of other intracranial or extracranial vessels. Patients with isolated MCA disease were divided into atherosclerotic and potentially cardioembolic, and the clinical, laboratory, and neuroradiological data analysed.

Results: Among the 850 consecutive patients with acute ischaemic stroke or transient ischaemic attack, $107(12.6 \%)$ met the criteria for isolated MCA disease (76 with atherosclerotic disease and 31 with a potential source of cardiac embolism). Total anterior circulation infarcts were more common and baseline NIHSS score was higher in potentially embolic occlusions than in atherosclerotic disease (each $p<0.001$ ). While cortical infarcts and territorial infarcts were more common in the potential embolism group $(p=0.028$ and $p<0.001$, respectively), subcortical border zone infarcts were more common in the atherosclerotic group $(p<0.001)$. Multiple regression analysis showed that border zone infarcts and mild stroke were independently associated with atherosclerotic MCA disease, while territorial and cortical infarcts were associated with potential cardiac embolic disease.

Conclusions: Clinical and neuroradiological characteristics can differentiate isolated atherosclerotic MCA disease from MCA disease associated with potential sources of cardiac embolism, and may reflect the differences in underlying pathogenesis.
\end{abstract}

1 olated atherosclerotic lesions in large intracranial arteries, especially the middle cerebral artery (MCA), have been considered rare causes of ischaemic stroke in the white population; however, previous studies have shown that they are common in Asians. ${ }^{1-5}$ Atherosclerotic MCA disease presents morphologically as stenosis or occlusion on angiography, and can mimic embolic MCA lesions from either the heart or the internal carotid artery. In unselected series, about one third of the cases of MCA occlusion are attributed to emboli from the heart. ${ }^{5}{ }^{6}$ The fate of embolic MCA occlusions can be varied. ${ }^{78}$ Although the rate of spontaneous recanalisation is dependent on the character of the embolus and may reflect differences in study design, ${ }^{9}$ most embolic occlusions recanalise completely. Thus, if repeat angiography shows that an MCA lesion has resolved a diagnosis of embolism could be inferred, but if there is permanent occlusion or partial recanalisation it is difficult to differentiate an embolic lesion from in situ thrombotic lesions such as thrombotic occlusion or incompletely developed atheroma. ${ }^{10}$ Furthermore, in situations where recanalisation has not yet fully occurred, especially when seen for the first time or when intracranial atherosclerosis coexists with cardiac disease, the distinction between cardioembolic and atherosclerotic MCA disease is more difficult.

In contrast to cardioembolic MCA disease, the pathogenesis of atherosclerotic MCA disease includes thrombosis leading to complete occlusion, thromboembolism, haemodynamic compromise, or combinations of these factors. ${ }^{511}$ Assuming that the differing pathogenesis of MCA disease may be reflected in specific clinical and neuroradiological features, we have studied a consecutive series of patients with isolated MCA disease to determine whether atherosclerotic MCA disease can be distinguished by such features from isolated MCA disease accompanied by clinical evidence of cardioembolism.

\section{METHODS}

From September 2000 to May 2003, we studied retrospectively consecutive patients with isolated MCA disease who were admitted to Ajou University hospital within seven days of symptom onset. Isolated MCA disease was defined as a unilateral MCA stenosis of $>50 \%$ or occlusion on magnetic resonance angiography (MRA) or transfemoral conventional angiography (TFCA) without significant involvement of other intracranial or extracranial arteries on the diagnostic workup. The vascular neuroimaging workup consisted of TFCA, MRA (with carotid bifurcation imaging), or MRA with carotid Doppler. All patients underwent transthoracic echocardiography. Depending on whether there was clinical evidence of cardiogenic embolism, we divided isolated MCA disease into atherosclerotic disease and potentially cardioembolic disease. Isolated MCA diseases accompanying contralateral hemispheric or posterior circulation spotty lesions on diffusion weighted imaging (DWI) were excluded. In contrast to the description of cardioembolism used in the TOAST classification, ${ }^{12}$ MCA disease with a potential source of cardiac embolism in the present study included any subcortical

\footnotetext{
Abbreviations: CS, combined subcortical infarct; DP, deep perforator infarct; DWI, diffusion weighted imaging; $\mathrm{EB}$, external border zone; $\mathrm{IB}$, internal border zone; MCA, middle cerebral artery; MRA, magnetic resonance angiography; NIHSS, National Institutes of Health stroke scale; SP, superficial perforator infarct; TFCA, transfemoral conventional angiography; TOAST, time resolved optical absorption and scattering tomography
} 
infarcts regardless of their size or the clinical presentation of lacunes. Potential sources of cardiogenic embolism were as follows: recent myocardial infarction (less than three weeks before), known atrial fibrillation with or without mural thrombus, mitral stenosis or prosthetic valve, dilated cardiomyopathy, sick sinus syndrome, and acute bacterial endocarditis. For the sake of an exact aetiological classification, patients who did not have a full workup or those who had two or more aetiologies were excluded from the study. We also excluded patients with bilateral isolated MCA stenosis exceeding $50 \%(n=18)$.

Magnetic resonance imaging (MRI) was done within one week of the onset of symptoms (mean 2.5 days; range 1 to 7 ). We undertook a comparative analysis to determine whether the clinical and radiological features of atherosclerotic MCA disease could be distinguished from those of MCA disease with a potential source of cardiac embolism.

We reviewed the medical history, neurological examination findings, and laboratory test results. According to the clinical stroke syndrome, we divided patients into lacunar, partial anterior circulation, and total anterior circulation infarcts. ${ }^{13}$ The vascular risk factors were identified as follows:

- hypertension: use of antihypertensive agents, or systolic blood pressure $\geqslant 160 \mathrm{~mm} \mathrm{Hg}$, or diastolic blood pressure $\geqslant 95 \mathrm{~mm} \mathrm{Hg}$ on admission;

- diabetes mellitus: use of oral hypoglycaemic agents or glycosylated haemoglobin $>6.4 \%$;

- hypercholesterolaemia: use of antihyperlipidaemic agents or serum cholesterol level $>220 \mathrm{mg} / \mathrm{dl}$;

- smoking: any cigarette usage within the 28 days preceding the index stroke.

All patients underwent DWI. MRA was done on a $1.5 \mathrm{~T}$ machine (GE, Signa, Advantage). The diagnosis of infarcts on the MCA territory was made with the use of previously published templates (fig 1). ${ }^{14-16}$ MCA lesions on DWI were individually classified as subcortical, cortical (involvement of only one M2 branch of MCA), and territorial infarcts (involvement of more than one M2 branch of the MCA or MCA stem involvement). ${ }^{7}$ According to the topographical classification suggested by Bogousslavsky and Regli, subcortical infarcts were further subdivided into deep perforator (DP), border zone including either internal or external border zone infarcts, superficial perforator (SP), and combined subcortical (CS) infarcts. ${ }^{15}$ Using DWI, we also analysed spotty lesions in the cortical region concomitantly. Haemorrhagic transformation was assessed using baseline computed tomography (CT), with further CT or MRI within one week after symptom onset. Any areas of heterogeneous or homogeneous hypointense signal within the infarct area on T2 weighted imaging or DWI, and heterogeneous or homogeneous high signal on non-contrast CT were defined as haemorrhagic transformation. ${ }^{17} 18$

In addition, we recruited patients with acute MCA infarcts on DWI who had a potential source of cardioembolism without an occlusive MCA lesion on MRA, to determine whether the clinicoradiological features of MCA disease with a potential source of cardiac embolism could be distinguished from those of recanalised cardioembolic MCA infarcts. These patients were selected from the same study pool of isolated MCA disease and were defined as cases of potential cardioembolism without MCA disease.

We measured Ml stenosis on MRA or TFCA by previously suggested methods. ${ }^{19}$ The Ml stenosis was calculated according to the residual lumen diameter measured at the site of maximum narrowing and the diameter of the adjacent normal vessel, from which the percentage stenosis was calculated. The degree of stenosis was graded as follows: low degree of stenosis (50-74\%); high degree of stenosis (7599\%); and occlusion. These measurements were made independently by two of us (LPH and OSH), using a hand held caliper; an acceptable level of interobserver agreement in interpreting angiographic stenosis was observed $(\kappa=0.853)$. Because of poor visualisation of the lenticulostriatal artery on MRA, the location of MCA disease was roughly divided into proximal and distal halves of Ml.

\section{Statistics}

Statistical analyses were undertaken using a commercially available software package (SPSS, version 10.0). We used $\chi^{2}$ and $t$ tests to compare clinical features and stroke subtype with respect to aetiology when the variables were categorical and continuous, respectively. Variables were considered for multivariate analysis if they had a probability $(\mathrm{p})$ value of $\leqslant 0.1$ in univariate analysis. Logistic regression analysis using the backward stepwise method was applied to look for independent predictors of atherosclerotic MCA disease and MCA occlusion with a potential source of cardiac embolism. Independent variables were sex, age, risk factors, mild stroke (NIHSS score ${ }^{20}<6$ ), severe stroke (NIHSS score $\geqslant 6)$, stroke syndrome, and neuroradiological topography. Results are given as odds ratios (OR) as estimates of relative risk with $95 \%$ confidence intervals (CI). A p value of less than 0.05 was used to determine a statistically significant difference.

\section{RESULTS}

Among the 850 consecutive patients with acute ischaemic stroke or transient ischaemic attacks, 107 (12.6\%) met the criteria for isolated MCA disease. Among these 107 patients, 76 were classified as atherosclerotic and 31 as potentially cardioembolic. Of the latter, 20 had atrial fibrillation, five had valvar heart disease, three had recent myocardial infarction, two had dilated cardiomyopathy, and one had sick sinus syndrome. Details of the clinical features in the two groups are given in table 1 . Twelve patients with atherosclerotic MCA disease (15.8\%) and one with potentially cardioembolic MCA disease $(3.2 \%)$ presented with a clinical diagnosis of transient ischaemic attacks (TIA).

Clinically, while total anterior circulation infarcts were more common in the potential cardiac embolism group $(\mathrm{p}<0.001)$, lacunar and partial anterior circulation infarcts were more often observed in the atherosclerotic group $(\mathrm{p}=0.012$ and 0.004 , respectively). Patients with atherosclerotic MCA disease were younger $(p=0.006)$ and more often had hypertension and diabetes $(p=0.024$ and 0.038 , respectively) than patients with MCA disease with a potential source of cardiac embolism. Serum triglyceride was higher in the atherosclerotic group than in the potential embolism group (mean (SD): $2.00 \quad(1.92) \quad v \quad 1.15 \quad(0.55) \quad \mathrm{mmol} / \mathrm{l}$, $\mathrm{p}=0.002)$. The degree of the initial neurological deficit, defined as baseline NIHSS score, was more severe in the potential embolism group than in the atherosclerotic group (9.7 (4.7) $v 4.6$ (3.9), $\mathrm{p}<0.001)$. The potential embolism group arrived more rapidly at hospital and more often presented with a sudden onset of stroke than the atherosclerotic group $(\mathrm{p}=0.002$ and 0.001 , respectively). No significant differences in sex, smoking, or other laboratory data (initial glucose, total cholesterol, low density lipoprotein cholesterol, high density lipoprotein cholesterol, packed cell volume) were detected between the two groups.

TFCA was done in 22 patients with atherosclerotic MCA disease (five with low degree stenosis, 10 with high degree stenosis, and seven with occlusion) and in three patients with potentially cardioembolic MCA disease (all had MCA occlusions). No patients with potentially cardioembolic MCA 
A

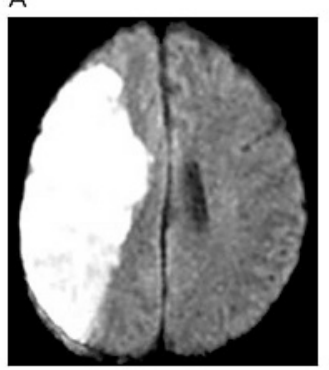

C

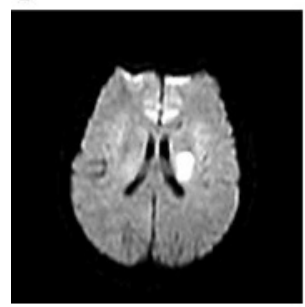

DP

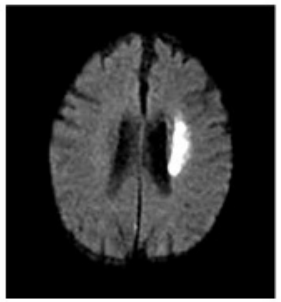

IB
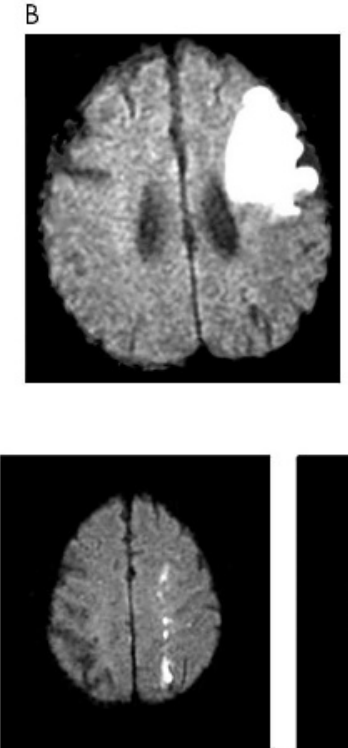

$E B$

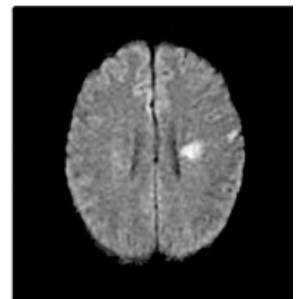

SP

Figure 1 Definition of topographic patterns using diffusion weighted magnetic resonance imaging. Territorial infarcts $(A)$ showing involvement of more than one M2 branch of the middle cerebral artery (MCA), or MCA stem involvement and cortical infarcts (B), showing involvement of only one M2 branch of the MCA. Subcortical infarcts (C) are subdivided into deep perforator (DP) infarcts, border zone infarcts including either internal (IB) or external border zone (EB) infarcts, and superficial perforator (SP) infarcts.

Table 1 Demographic features of patients with middle cerebral artery occlusions

\begin{tabular}{|c|c|c|c|c|c|}
\hline Variable & $\begin{array}{l}\text { AS-MCA } \\
(n=76)\end{array}$ & $p$ Value & $\begin{array}{l}\text { PSCE-MCA } \\
(n=31)\end{array}$ & $p$ Value & $\begin{array}{l}\text { PSCE without MCA } \\
(n=30)\end{array}$ \\
\hline Male sex & $39(52.0)$ & NS & $18(58.1)$ & NS & $16(53.3)$ \\
\hline Age (years)* & $58(13)$ & $0.006 \dagger$ & $66(14)$ & NS & $64(11)$ \\
\hline \multicolumn{6}{|l|}{ Risk factors } \\
\hline Hypertension & $51(61.8)$ & $0.024 \ddagger$ & $13(43.3)$ & NS & $12(40.0)$ \\
\hline Díabetes & 25 (33.3) & $0.038 \S$ & $4(13.3)$ & NS & $5(16.7)$ \\
\hline Smoking & $23(35.9)$ & NS & $9(30.0)$ & NS & $10(33.3)$ \\
\hline Blood glucose $(\mathrm{mmol} / \mathrm{l})^{*}$ & $8.73(4.28)$ & NS & $8.51(3.42)$ & NS & $8.73(3.33)$ \\
\hline $\begin{array}{l}\text { Total serum cholesterol } \\
(\mathrm{mmol} /)^{\star}\end{array}$ & $4.88(1.10)$ & NS & $4.61(1.07)$ & NS & $5.07(1.48)$ \\
\hline LDL (mmol/l)* & $2.86(0.92)$ & NS & $2.93(0.87)$ & NS & $2.62(0.99)$ \\
\hline $\mathrm{HDL}(\mathrm{mg} / \mathrm{dll})^{*}$ & $1.19(0.29)$ & NS & $1.24(0.24$ & NS & $1.28(0.33)$ \\
\hline TG $(\mathrm{mg} / \mathrm{dll})^{*}$ & 2.00 (1.92) & $0.002 \dagger$ & $1.15(0.55)$ & NS & $1.28(0.47)$ \\
\hline PCV $(\%)^{*}$ & $39.8(6.7)$ & NS & $40.3(5.8)$ & NS & $41.6(6.1)$ \\
\hline \multicolumn{6}{|l|}{ Stroke syndrome } \\
\hline Lacuna & $31(48.4)$ & $0.012 \ddagger$ & $6(20.0)$ & NS & $2(6.7)$ \\
\hline Partial & $25(39.1)$ & $0.004 \S$ & $3(10.0)$ & $<0.001 \S$ & $22(73.3)$ \\
\hline Total & $8(12.5)$ & $<0.001 \ddagger$ & $21(70.0)$ & $<0.001 \S$ & $5(16.7)$ \\
\hline Initial NIHSS score* & $4.6(3.9)$ & $<0.001 \dagger$ & $9.7(4.7)$ & $<0.001 \dagger$ & $5.2(3.6)$ \\
\hline \multicolumn{6}{|l|}{ Time from onset to } \\
\hline $\begin{array}{l}\text { hospital (h) } \\
\text { Abrupt onset }\end{array}$ & $37(48.7)$ & $0.001 \ddagger$ & $26(83.8)$ & NS & $26(86.6)$ \\
\hline Site of MCA lesion & & $0.008 \ddagger$ & & & \\
\hline Proximal & $59(77.6)$ & & $16(51.6)$ & - & - \\
\hline Distal & 17 (22.4) & & $15(48.4)$ & - & - \\
\hline \multicolumn{6}{|l|}{ Degree of stenosis } \\
\hline Low degree & $19(25.0)$ & NS & $6(19.4)$ & - & - \\
\hline High degree & $32(32.1)$ & NS & $6(19.4)$ & - & - \\
\hline Occlusion & $25(32.9)$ & $0.007 \ddagger$ & $19(61.2)$ & - & - \\
\hline
\end{tabular}

Values are $\mathrm{n}(\%)$ or *mean (SD).

AS-MCA, atherosclerotic middle cerebral artery disease; $\mathrm{HDL}$, high density lipoprotein cholesterol; LDL, low density lipoprotein cholesterol; MCA, middle cerebral artery disease; NIHSS, National Institutes of Health stroke scale; Partial, partial anterior circulation infarct; PCV, packed cell volume; PSCE-MCA, middle cerebral artery disease with potential source of cardioembolism; TG, triglycerides; Total, total anterior circulation infarct. †Mann-Whitney test, $\ddagger$ Pearson $\chi^{2}$ test, SFischer's exact test. 
disease had collaterals from the posterior or anterior cerebral artery, whereas 14 patients $(63.6 \%)$ with atherosclerotic MCA disease diseases had good collaterals on TFCA. Based on angiographic characteristics, lesions of the MCA were more proximally located in the atherosclerotic group than in the potential embolism group $(77.6 \% \vee 51.6 \%, p=0.008)$ and complete MCA occlusion was more common in the potential embolism group than in the atherosclerotic group $(61.2 \% \mathrm{v}$ $32.9 \%, \mathrm{p}=0.007)$.

Excluding 12 patients with atherosclerotic MCA disease who presented with TIA without fresh lesions on DWI, we made a comparative analysis of radiological patterns between patients with atherosclerotic MCA disease $(n=64)$ and patients with potentially cardioembolic MCA disease $(\mathrm{n}=31)$ (table 2). Neuroradiologically, subcortical infarcts were significantly more common in the atherosclerotic group than in the potential embolism group $(82.8 \% \vee 19.4 \%$, $\mathrm{p}<0.001)$. Individual analysis of subcortical infarcts showed that border zone infarcts were more common in the atherosclerotic group than in the potential embolism group $(p<0.001)$ and there was a trend for DP infarcts to be more common in the atherosclerotic group $(p=0.049)$. The border zone infarct group comprised 15 patients with internal border zone infarcts, six with external border zone infarcts, and three with combined internal and external border zone infarcts. Cortical infarcts and territorial infarcts, however, were significantly more common in MCA disease with a potential source of cardiac embolism $(p=0.028$ and $p<0.001$, respectively). Cortical spotty lesions on DWI did not differ between the two groups.

On multivariate logistic regression analysis, border zone infarcts (OR 13.4 (95\% CI, 1.5 to 118.3$), \mathrm{p}=0.019)$ and mild stroke (OR 9.2 (2.9 to 28.6)) were significant predictors of atherosclerotic MCA disease, while territorial infarcts (OR 52.9 (2.2 to 138.8), $\mathrm{p}=0.006$ ) and cortical infarcts (OR 9.6 (2.4 to 38.0$), p=0.001$ ) were independently associated with potentially cardioembolic MCA disease. Though not reaching statistical significance, DP infarcts (OR 3.8), cortical spotty lesions (OR 3.4), and SP infarcts (OR 2.1) tended to be associated with atherosclerotic MCA disease, and haemorrhagic transformation (OR 3.1) trend to be associated with potentially cardioembolic MCA disease.

The topographic patterns are schematically illustrated in fig 2 with respect to the degree of MCA stenosis. In MCA occlusion, potentially cardioembolic MCA diseases were often manifested by territorial infarcts, while atherosclerotic MCA disease was associated with border zone infarcts $(\mathrm{p}<0.001)$.

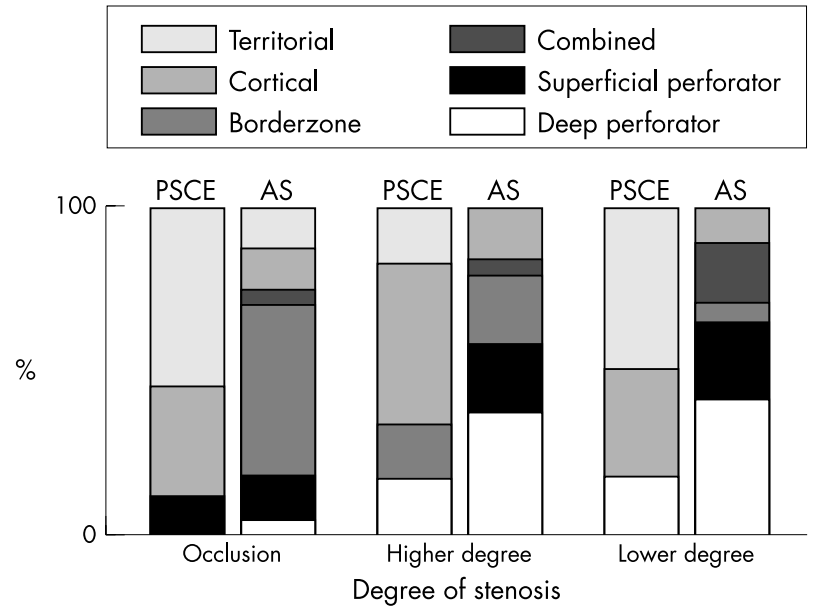

Figure 2 Differences in topography between atherosclerotic middle cerebral artery (MCA) disease and MCA disease with potential sources of cardioembolism, related to the degree of stenosis. AS, atherosclerotic MCA disease; PSCE, MCA disease with a potential source of cardioembolism.

In high degree stenosis, MCA disease with a potential source of cardiac embolism were related to cortical infarcts more often than atherosclerotic MCA disease $(p=0.04)$.

In a comparative analysis between the two groups of patients with potential sources of cardiac embolism (with and without occlusive MCA disease; tables 1 and 2), the group with MCA disease was more likely to have total anterior circulation infarcts and territorial infarcts $(p<0.001$ and $p=0.002$, respectively), while the group without MCA disease was more likely to have partial anterior circulation infarcts and cortical infarcts $(\mathrm{p}<0.001$ and $\mathrm{p}=0.011$, respectively). Among the patients with potential sources of cardiac embolism, the initial NIHSS score was higher in those with MCA occlusive disease than in those without $(9.7$ (4.7) $v$ 5.2 (3.6); $\mathrm{p}<0.001$ ); haemorrhagic transformation was more common in the group without MCA occlusive disease, but the difference was not statistically significant. The average time from onset of stroke to TFCA or MRA was not significantly different between the two groups.

\section{DISCUSSION}

Studies on spontaneous recanalisation after cardioembolic stroke show that, although most MCA occlusions

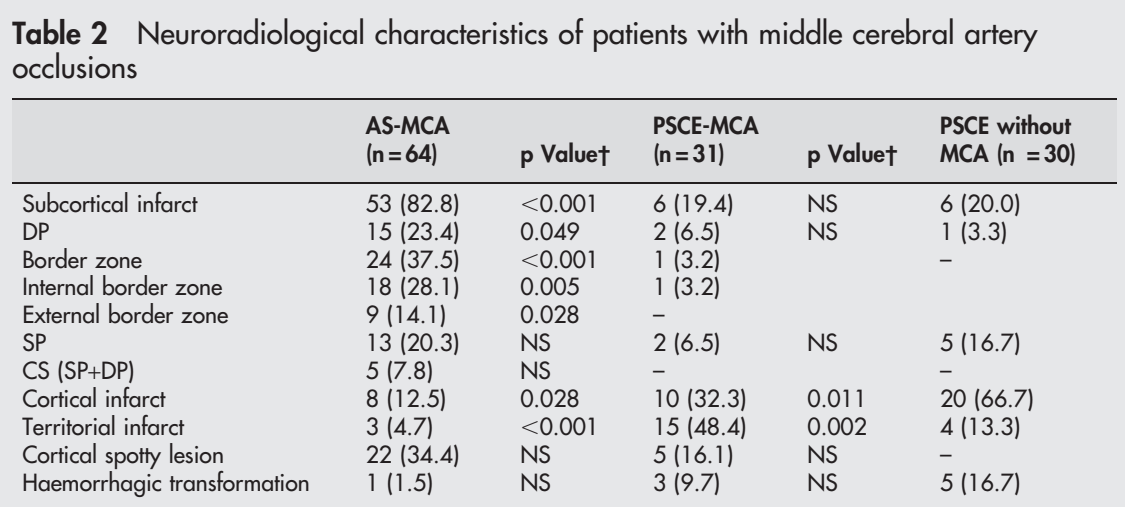

Values are $\mathrm{n}(\%)$.

Together with internal border zone infarct (3), combined subcortical infarct (3), and superficial perforator infarct (1).

†Fischer's exact test.

AS-MCA, atherosclerotic middle cerebral artery disease; CS, combined subcortical infarct; DP, deep perforator infarct; MCA, middle cerebral artery disease; PSCE-MCA, middle cerebral artery disease with potential source of cardioembolism; SP, superficial perforator infarct. 
recanalise spontaneously in the first days, up to $30 \%$ remain occluded even up to seven days after the event. ${ }^{78}$ However, it is known that angiographic characteristics are unreliable in distinguishing between atherosclerotic and cardioembolic MCA lesions. ${ }^{10}$ In present study, about a half the patients with presumed cardioembolic infarcts in the MCA territory showed either MCA stenosis or occlusion on angiography, which mimicked the lesions of atherosclerotic MCA disease. As well as describing the vascular characteristics of spontaneous recanalisation, our study focused on certain clinical and neuroradiological characteristics that were helpful in differentiating between atherosclerotic and cardioembolic MCA disease.

Clinically, patients with potentially cardioembolic MCA disease were likely to have total anterior circulation infarcts, and to have a higher baseline NIHSS score than patients with atherosclerotic MCA disease. On the other hand, the atherosclerotic group presented with mild neurological deficits, such as lacunes or partial anterior circulation infarcts. This difference in neurological deficits between the two groups may explain the more rapid arrival in hospital of patients with potentially cardioembolic MCA disease. The increased prevalence of atrial fibrillation with age may account for the older mean age of patients with potentially cardioembolic MCA disease. On the basis of the topographic patterns, the atherosclerotic group was more likely to present with subcortical infarcts, while the potentially cardioembolic group was more likely to present with cortical or territorial infarcts, which were well correlated with the clinical presentation. These clinical and topographical features could be ascribed to differences in the adequacy of collateral perfusion between the two groups. ${ }^{21}$ There may be well developed collaterals in pre-existing and long standing atherosclerotic MCA disease, whereas collaterals tend to be inadequate in MCA occlusion caused by embolic disease.

In subgroup analysis of subcortical infarcts, although DP infarcts were often associated with atherosclerotic MCA disease-in accordance with our previous report that around $40 \%$ of deep striatocapsular infarcts were associated with atherosclerotic MCA disease ${ }^{22}$-border zone infarcts were the sole independent predictor of atherosclerotic MCA disease on multivariate analysis. Internal border zone (IB) infarcts accounted for about $75 \%$ of patients with border zone infarcts in the present study. In previous studies it was found that patients with IB infarcts had a higher frequency of severe carotid artery stenosis or occlusion, ${ }^{23}{ }^{24}$ and a haemodynamic aetiology was suggested; this is indirectly supported by PET and SPECT studies. ${ }^{25}{ }^{26}$ Angeloni et al reported that IB infarcts may result from isolated MCA occlusions, ${ }^{27}$ and our data show that IB infarcts can result from isolated atherosclerotic MCA disease without carotid artery stenosis or occlusion. IB infarcts may serve as a neuroradiological clue to differentiate isolated atherosclerotic MCA disease from potentially embolic MCA disease. On the other hand, the incidence of SP infarcts located in the most superficial part of the corona radiata and the long association fasciculi $^{28}{ }^{29}$ did not differ between the two groups. Although the mechanism of SP infarcts is not settled, Yonemura et al recently suggested that these infarcts are associated with both emboligenic heart disease and large artery disease, ${ }^{30}$ which explains why there was no difference in SP infarcts between the two groups in our study. This is further supported by the finding that the incidence of cortical spotty lesion detectable by DWI-known to be small embolic signals either from large arteries or the heart ${ }^{31}$-did not differ between the two groups.

On angiography, MCA lesions in the atherosclerotic group were located in the more proximal part of the artery, reflecting the similar mechanism in extracranial atherosclerosis, where the lesions are frequently observed in regions of arterial bifurcation. In our comparison between the degree of stenosis and neuroradiological topography, MCA occlusion or high degree stenosis had different clinical manifestation depending on the pathogenesis (fig 2); the atherosclerotic group often presented with border zone infarcts, whereas the potentially cardioembolic group mainly presented with cortical or territorial infarcts. These findings are in agreement with previous reports emphasising the importance of collateral circulatory pathways and collateral perfusion in determining infarct pattern and size..$^{27} 3233$ In our comparison of patients with potential sources of cardiogenic emboli who did and did not have occlusive MCA disease, the pattern of cortical involvement was similar in the two groups, suggesting that they shared the common underlying pathogenesis of cardioembolism; however, the patients with occlusive MCA disease had more severe neurological deficits and a radiologically more extensive infarct area, in agreement with previous reports that early recanalisation of embolic MCA occlusions has a favourable impact on infarct size and clinical outcome. ${ }^{78}$

The differentiation between cardioembolic and atherosclerotic MCA disease may have clinical implications for early anticoagulant treatment in cardioembolic stroke. Although this subject remains under debate, it is known that recurrent cardioembolic strokes mostly occur in the early stage after infarction. ${ }^{34}$ However, the mechanism of atherosclerotic MCA disease is known to be related to thromboembolism or haemodynamic compromise, and thus an individual strategy is required depending on the pathogenesis.

It may be unwise to extrapolate our findings relating to MCA disease associated with a potential source of cardiac embolism to definite cardioembolic MCA disease without acquiring data from repeated vascular neuroimaging. This reflects the controversy over cardiogenic embolism as a cause of subcortical infarcts. Despite the fact that the association of DP infarcts with cardioembolism is still under debate, ${ }^{35-38}$ recent reports suggest that the embolic contribution to the pathogenesis of border zone and SP infarcts is more common than expected..$^{30}{ }^{39}$ Furthermore, in our study one patient with potentially cardioembolic MCA disease who had DP infarcts showed complete recanalisation of the MCA at follow up angiography, suggesting a cardioembolic mechanism. To resolve this issue, further study with follow up vascular neuroimaging is warranted.

\section{Conclusions}

In this study, infarcts caused by underlying haemodynamic compromise were related to atherosclerotic middle cerebral artery disease, while tandem artery infarcts caused by large emboli were more common when there was MCA disease with potential sources of cardioembolism. Our study also suggests that the clinical and neuroradiological characteristics of the cerebral lesions reflect the differences in underlying pathogenesis, and are helpful in differentiating between isolated atherosclerotic MCA disease and MCA disease with a potential source of cardiac embolism.

\section{Authors' affiliations}

P H Lee, S H Oh, O Y Bang, I S Joo, K Huh, Department of Neurology, School of Medicine, Ajou University, South Korea

Competing interests: none declared

\section{REFERENCES}

1 Gorelick PB, Caplan LR, Hier DB, et al. Racial differences in the distribution of anterior circulation occlusive disease. Neurology 1984;34:54-9.

2 Caplan LR, Gorelick PB, Hier DB. Race, sex and occlusive cerebrovascular disease: a review. Stroke 1986;17:648-55. 
3 Leung SY, Ng TH, Yuen ST, et al. Pattern of cerebral atherosclerosis in Hong Kong Chinese: severity in intracranial and extracranial vessels. Stroke 1993;24:779-86

4 Wong KS, Huang YN, Gao S, et al. Intracranial stenosis in Chinese patients with acute stroke. Neurology 1998;50:812-13.

5 Bogousslavsky J, Barnett HJM, Fox AJ, et al. Atherosclerotic disease of the middle cerebral artery. Stroke 1986;17:1112-20.

6 Moulin DE, Lo R, Chiang J, et al. Prognosis in middle cerebral artery occlusion. Stroke 1985; 16:282-4.

7 Ringelstein EB, Biniek R, Weiller C, et al. Type and extent of hemispheric brain infarctions and clinical outcome in early and delayed middle cerebral artery recanalization. Neurology 1992;42:289-98.

8 Molina CA, Montaner J, Abilleira S, et al. Timing of spontaneous recanalization and risk of hemorrhagic transformation in acute cardioembolic stroke. Stroke 2001;32:1079-84.

9 Kassem-Moussa H, Graffagnino C. Nonocclusion and spontaneous recanalization rates in acute ischemic stroke: a review of cerebral angiography studies. Arch Neurol 2002;59:1870-3.

10 Mohr JP, Lazar RM, Marshall RS, et al. Middle cerebral artery disease. In: Barnett HRM, Mohr JP, Stein BM, et al, eds. Stroke: pathophysiology, diagnosis and management, 3rd ed. New York: Churchill Livingstone, 1998:427-79.

11 Wong KS, Gao S, Chan YL, et al. Mechanisms of acute cerebral infarctions in patients with middle cerebral artery stenosis: a diffusion-weighted imaging and microemboli monitoring study. Ann Neurol 2002;52:74-81.

12 Adams HP, Bendixen BH, Kappelle $\sqcup$, et al. Classification of subtype of acute ischemic stroke: definitions for use in a multicenter clinical trial. Stroke 1993;24:35-41.

13 Bamford J, Sandercock P, Dennis M, et al. Classification and natural history of clinically identifiable subtypes of cerebral infarction. Lancet 1991;337:1521-6.

14 Tatu L, Moulin T, Bogousslavsky J, et al. Arterial territories of the human brain: cerebral hemispheres. Neurology 1998;50:1699-708.

15 Bogousslavsky J, Regli F. Centrum ovale infarcts: subcortical infarction in the superficial territory of the middle cerebral artery. Neurology 1992:42:1992-8.

16 van der Zwan A, Hillen B, Tulleken CAF, et al. Variability of the territories of the major cerebral arteries. J Neurosurg 1992;77:927-40.

17 Tong DC, Adami A, Moseley ME, et al. Prediction of hemorrhagic transformation following acute stroke: role of diffusion- and perfusionweighted magnetic resonance imaging. Arch Neurol 2001;58:587-93.

18 Fiorelli $M$, Bastianello $S$, von Kummer $R$, et al. Hemorrhagic transformation within 36 hours of a cerebral infarct: relationships with early clinical deterioration and 3-month outcome in the European Cooperative Acute Stroke Study I (ECASS I) cohort. Stroke 1999;30:2280-4.

19 Samuels OB, Joseph GJ, Lynn MJ, et al. A standardized method for measuring intracranial arterial stenosis. Am J Neuroradiol 2000;21:643-6.

20 Lyden P, Brott T, Tilley B, et al. Improved reliability of the NIH Stroke Scale using video training: NINDS TPA Stroke Study Group. Stroke 1994;25:2220-6.
21 Timsit SG, Sacco RL, Mohr JP, et al. Brain infarction severity differs according to cardiac or arterial embolic source. Neurology 1993;43:728-33.

22 Bang OY, Heo JH, Kim JY, et al. Middle cerebral artery stenosis is a major clinical determinant in striatocapsular small, deep infarction. Arch Neurol 2002:59:259-63.

23 Gandolfo C, Del Sette M, Finocchi C, et al. Internal borderzone infarction in patients with ischemic stroke. Cerebrovasc Dis 1998;8:255-8.

24 Del Sette M. Eliasziw M, Streifler JY, et al. Internal borderzone infarction: a marker for severe stenosis in patients with symptomatic internal carotid artery disease. Stroke 2000;31:631-6.

25 Yamauchi H, Fukuyama H, Yamaguchi S, et al. High-intensity area in the deep white matter indicating hemodynamic compromise in internal carotid artery occlusive disorders. Arch Neurol 1991:48:1067-71.

26 Moriwaki H, Matsumoto M, Hashikawa K, et al. Hemodynamic aspect of cerebral watershed infarction: assessment of perfusion reserve using iodine123-iodoamphetamine SPECT. J Nucl Med 1997;38:1556-62.

27 Angeloni U, Bozzao L, Fantozzi L, et al. Internal borderzone infarction following acute middle cerebral artery occlusion. Neurology 1990;40:1196-8.

28 Read SJ, Pettigrew L, Schimmel L, et al. White matter medullary infarcts: acute subcortical infarction in the centrum ovale. Cerebrovasc Dis 1998;8:289-95.

29 Donnan GA, Norrving B, Bamford JM, et al. Subcortical infarctions: classification and terminology. Cerebrovasc Dis 1993;3:248-51.

30 Yonemura K, Kimura K, Minematsu K, et al. Small centrum ovale infarcts on diffusion-weighted magnetic resonance imaging. Stroke 2002;33:1541-4.

31 Kimura K, Minematsu K, Koga M, et al. Microembolic Signals and Diffusionweighted MR imaging abnormalities in acute ischemic stroke. Am J Neuroradiol $2001 ; 22: 1037-42$.

32 Saito I, Segawa H, Shiokawa Y, et al. Middle cerebral artery occlusion: correlation of computed tomography and angiography with clinical outcome. Stroke 1987; 18:863-8.

33 Bozzao L, Fantozzi LM, Bastianello S, et al. Early collateral blood supply and late parenchymal brain damage in patients with middle cerebral artery occlusion. Stroke 1989;20:735-40.

34 Adams HP. Emergent use of anticoagulation for treatment of patients with ischemic stroke. Stroke 2002;33:856-61.

35 Cacciatore A, Russo LS. Lacunar infarction as an embolic complication of cardiac and arch angiography. Stroke 1991;22:1603-5.

36 Laloux P, Brucher JM. Lacunar infarctions due to cholesterol emboli. Stroke 1991;22:1440-4.

37 Mast H, Thompson JL, Voller H, et al. Cardiac sources of embolism in patients with pial artery infarcts and lacunar lesions. Stroke 1994;24:420-5.

38 Ringelstein EB, Koschorke S, Holling A, et al. Computed tomographic patterns of proven embolic brain infarctions. Ann Neurol 1989;26:759-65.

39 Caplan LR, Hennerici M. Impaired clearance of emboli (washout) is an important link between hypoperfusion, embolism, and ischemic stroke. Arch Neurol 1998;55:1475-82. 